\title{
INFERRING THE STRUCTURE OF THE SOLAR CORONA AND INNER HELIOSPHERE DURING THE MAUNDER MINIMUM USING GLOBAL THERMODYNAMIC MAGNETOHYDRODYNAMIC SIMULATIONS
}

\author{
Pete Riley $^{1}$, Roberto Lionello ${ }^{1}$, Jon A. Linker ${ }^{1}$, Ed Cliver ${ }^{2}$, Andre Balogh ${ }^{3}$, Jürg Beer ${ }^{4}$, Paul Charbonneau ${ }^{5}$, \\ Nancy Crooker ${ }^{6}$, Marc DeRosa ${ }^{7}$, Mike Lockwood ${ }^{8}$, Matt Owens ${ }^{8}$, Ken McCracken $^{9}$, Ilya Usoskin ${ }^{10}$, and S. Koutchmy ${ }^{11}$ \\ ${ }^{1}$ Predictive Science, 9990 Mesa Rim Road, Suite 170, San Diego, CA 92121, USA; pete@predsci.com, lionel@predsci.com, linkerj@predsci.com \\ ${ }^{2}$ National Solar Observatory, Sunspot, NM 88349, USA; ecliver@nso.edu \\ ${ }^{3}$ Imperial College, South Kensington Campus, Department of Physics, Huxley Building 6M68, London, SW7 2AZ, UK; a.balogh@ic.ac.uk \\ ${ }^{4}$ Surface Waters, Eawag, Ueberlandstrasse 133, P.O. Box 611, 8600 Duebendorf, Switzerland; Juerg.beer@eawag.ch \\ ${ }^{5}$ Département de Physique, Université de Montréal, C.P. 6128 Centre-Ville, Montréal, Qc, H3C-3J7, Canada; paulchar@astro.umontreal.ca \\ ${ }^{6}$ Center for Space Physics, Boston University, 725 Commonwealth Avenue, Boston, MA 02215, USA; crooker@bu.edu \\ ${ }^{7}$ Lockheed Martin Solar and Astrophysics Laboratory, 3251 Hanover St., B/252, Palo Alto, CA 94304, USA; derosa@1msal.com \\ ${ }^{8}$ University of Reading, Department of Meteorology, Reading, Berkshire, RG6 6BB, UK; lockwood@reading.ac.uk, m.j.owens@ reading.ac.uk \\ 9100 Mt. Jellore Lane, Woodlands, NSW, 2575, Australia; jellore@ hinet.au \\ ${ }^{10}$ Sodankyla Geophysical Observatory, FIN-90014, University of Oulu, Finland; ilya.usoskin@oulu.fi \\ ${ }^{11}$ Institut d'Astrophysique de Paris, CNRS and UPMC, Paris, France; koutchmy@iap.fr \\ Received 2014 September 29; accepted 2015 January 29; published 2015 March 30
}

\begin{abstract}
Observations of the Sun's corona during the space era have led to a picture of relatively constant, but cyclically varying solar output and structure. Longer-term, more indirect measurements, such as from ${ }^{10} \mathrm{Be}$, coupled by other albeit less reliable contemporaneous reports, however, suggest periods of significant departure from this standard. The Maunder Minimum was one such epoch where: (1) sunspots effectively disappeared for long intervals during a $70 \mathrm{yr}$ period; (2) eclipse observations suggested the distinct lack of a visible K-corona but possible appearance of the F-corona; (3) reports of aurora were notably reduced; and (4) cosmic ray intensities at Earth were inferred to be substantially higher. Using a global thermodynamic MHD model, we have constructed a range of possible coronal configurations for the Maunder Minimum period and compared their predictions with these limited observational constraints. We conclude that the most likely state of the corona during - at least—-the later portion of the Maunder Minimum was not merely that of the 2008/2009 solar minimum, as has been suggested recently, but rather a state devoid of any large-scale structure, driven by a photospheric field composed of only ephemeral regions, and likely substantially reduced in strength. Moreover, we suggest that the Sun evolved from a 2008/2009-like configuration at the start of the Maunder Minimum toward an ephemeral-only configuration by the end of it, supporting a prediction that we may be on the cusp of a new grand solar minimum.
\end{abstract}

Key words: Sun: activity - Sun: corona - Sun: heliosphere - Sun: magnetic fields - Sun: photosphere - sunspots

\section{INTRODUCTION}

The "Maunder Minimum" is a period of time between approximately 1645 and 1715 when the observed number of sunspots all but disappeared (Eddy 1976). Although it can be argued how accurate the sunspot record was during this interval, the low numbers cannot be due to a lack of observations; a number of well-known astronomers, including Giovanni Domenico Cassini, regularly made observations of the Sun during this time. During the same period, as we will discuss in more detail below, the number of aurora decreased, cosmic ray fluxes increased, and the Sun's corona apparently lost its visible structure.

Broadly speaking, we can differentiate between two distinct ideas for the state of the solar corona during the Maunder Minimum. The first, and original idea was of a corona that was radically different from what we observe today (Eddy 1976; Parker 1976; Suess 1979). The second, and currently more favored interpretation is of a corona that was not significantly different than the one observed during the recent and somewhat unique solar minimum of 2008/2009 (e.g., Svalgaard \& Cliver 2007).

In his landmark paper, Eddy (1976) reviewed an extensive range of available data associated with this time period, including auroral records, sunspots, carbon-14 records, and eclipse observations. From the (i) prolonged absence of sunspots; (ii) reduction in aurora reports; (iii) decrease in ${ }^{14} \mathrm{C}$ (suggesting a significant increase in cosmic ray flux hitting the Earth); and (iv) absence of any structured corona during eclipses, he inferred that, to manifest such phenomena, the solar corona must have existed in a unique configuration. He suggested that "the solar wind would have blown steadily and isotropically, and possibly at gale force, since high-speed streams of solar wind are associated with the absence of closed structure in the solar corona." He concluded that, based on eclipse observations, there likely was not any K-corona present, and that, in fact, what was observed could have been from dustscattered light (i.e., the F-corona). When asked about the Maunder Minimum, Parker (1976) suggested "In view of the absence of a white light corona, we may conjecture whether the Sun was entirely shrouded in a coronal hole, yielding a fast steady solar wind, or whether there simply was no solar wind at all. I would guess the former, but I know of no way to prove the answer." Suess (1979) expanded on these views: "Firstly, C-14 data indicate an enhanced cosmic ray intensity, with the conclusion that the interplanetary magnetic field was smooth and perhaps of low intensity. Secondly, the apparent absence of a corona during eclipses requires low coronal density, suggesting an absence of closed magnetic loops. Thirdly, the absence of sunspots eliminates the possibility of a solar maximum type of corona of low emission intensity and implies a low large-scale photospheric field intensity. Finally, the 
absence of mid-latitude aurorae implies either that the solar wind speed or the IMF intensity, or both, were low and not irregular."

More recently, the idea that the Maunder Minimum was radically different than anything witnessed during the space era has come to be replaced with the idea that the recent, and arguably unique (at least on the timescale of a century) solar minimum of 2008/2009 provided an accurate proxy for Maunder Minimum conditions. This perspective has been built up on two primary fronts. First, Svalgaard \& Cliver (2007) proposed that there appears to be minimum value $(\sim 4.6 \mathrm{nT})$ in the strength of the interplanetary magnetic field. It is important to recognize, however, that the proposal by Svalgaard \& Cliver (2007) was a hypothesis rather than a robust, empirically determined result and is based on geomagnetic observations from 1835 onwards, an interval that includes neither the Dalton nor the Maunder minima. Other authors have argued that the occurrence probability of open solar flux (OSF) and near-Earth IMF becomes smaller at lower values (e.g., Lockwood et al. 2014a) and hence the lowest value seen in a given interval does not, in itself, set a physical limit. In addition, it has been pointed out that arguments supporting a floor are implicitly based on the concept that the OSF and near-Earth IMF depend only on the simultaneous sunspot number, whereas successful continuity modeling of OSF is based on the idea that the prior history of sunspot numbers is also important (Lockwood et al. 2014b). Although the strength of the near-Earth IMF has apparently returned to the roughly similar values since the start of the space era, there is no guarantee that this must remain so; in fact, the precise value of this "floor" had to be lowered (Cliver \& Ling 2011) as we witnessed the minimum of 2008/2009 (Crooker \& Owens 2010; Cliver \& Ling 2011). Second, Schrijver et al. (2011) argued that there exists a minimum state of solar magnetic activity, associated with the presence of small-scale ephemeral regions. They concluded that "the best estimate of magnetic activity... for the least-active Maunder Minimum phases appears to be provided by direct measurements in 2008-2009," although they acknowledged that the presence of a large-scale dipolar field may not be representative of the deepest portions of the Maunder Minimum. Cliver (2012) noted that a minimal solar activity state such as that characterized by the small-scale ephemeral regions of Schrijver et al. (2011) is a requirement for a floor in the IMF. Finally, in perhaps what could be described as a compromise between these two extreme views, Wang \& Sheeley (2013) presented a solution where the photospheric field consisted of ephemeral regions with a reduced, but not absent global dipole. They reasoned that a Sun consisting exclusively of ephemeral regions would generate no IMF, in conflict with the apparent continuation of the solar cycle (albeit much reduced) during the Maunder Minimum (e.g., Ribes \& Nesme-Ribes 1993; Cliver et al. 1998; McCracken et al. 2013).

Most recently, McCracken \& Beer (2014) reviewed the cosmogenic records $\left({ }^{10} \mathrm{Be}\right.$ and $\left.{ }^{14} \mathrm{C}\right)$, concluding that inferred cosmic ray intensities during the Maunder Minimum were significantly greater than those during the 2008/2009 minimum, and, in addition, the inferred heliospheric magnetic field $(\sim 2 \mathrm{nT})$ was half the value measured in 2009 by in situ spacecraft.

In this study, we apply a global MHD model to infer the most likely configuration of the solar corona (and by extension, the inner heliosphere) that is consistent with the observations associated with the Maunder Minimum. In Section 2, we review, re-analyze, and extend previous studies of the limited, and often indirect observations of the period between 1645 and 1715. In Section 3, we introduce a set of possible configurations of the photospheric magnetic field that may have existed during the Maunder Minimum, and use them to drive MHD simulations. In Section 4 we describe our MHD formalism, paying particular attention to the unique aspects that make it applicable and relevant for studying the Maunder Minimum. We then describe the model results and relate them to the observations, allowing us to refute some configurations, while finding support for others. Finally, in Section 5 we summarize the main points of this study, and discuss the implications in terms of both understanding the Maunder Minimum and other periods of inactivity, and the likelihood of future grand minima.

\section{OBSERVATIONS DURING THE MAUNDER MINIMUM}

Although a number of studies since J. Eddy's landmark paper (Eddy 1976) have revised and refined our interpretation of the available but limited observations associated with the Maunder Minimum, it is worth reviewing them here, both to point out where they led to definitive inferences and where they remain ambiguous. Additionally, it affords us an opportunity to offer our own interpretation.

The observations we consider here are: (1) the sunspot time series; (2) aurora reports; (3) cosmic ray fluxes as inferred from ${ }^{10} \mathrm{Be}$ and ${ }^{14} \mathrm{C}$ records; and (4) eclipse observations. Figure 1 summarizes the first three of these records stretching back from near-present day to the Maunder Minimum. In the following subsections, we consider each in more detail.

\subsection{Sunspot Observations}

Figure 1(a) summarizes the sunspot record back in time to 1610 (Lockwood et al. 2014a, 2014b; Lockwood \& Owens 2014). This is the parameter that originally defined the Maunder Minimum, although the numbers themselves have undergone significant revision since they were first presented by G. Spörer and E. W. Maunder in the late 19th century (Sporer 1887; Maunder 1894). As a practical definition, we follow the consensus of identifying the Maunder Minimum as the time period between $\sim 1645$ and $\sim 1700-1715$ when sunspots all but disappeared. It is important to emphasize, however, that they did not disappear entirely; there were isolated observations of sunspots throughout this period.

The sunspot record is not without error or uncertainty, however. First, we must confront the claim that the reduction in sunspot number (SSN) was not due to processes at the Sun, but from a lack of observations. Hoyt \& Schatten (1996) examined how well sunspots were observed during the Maunder Minimum, concluding that $68 \% \pm 7 \%$ of the days were observed. Therefore, the paucity of sunspots was due to them not being observed, not from there being no observations. Second, the apparent lack of sunspots may have been caused by atmospheric effects, such as the presence of volcanic ash masking the sunspots or increasing the threshold for detection. Third, revisions to the sunspot record have been proposed over the years (e.g., Hoyt \& Schatten 1998; Clette et al. 2014; Lockwood et al. 2014a). Although this could have a quantitative effect on the record, it is unlikely to change the basic, qualitative profile shown in Figure 1(a). 

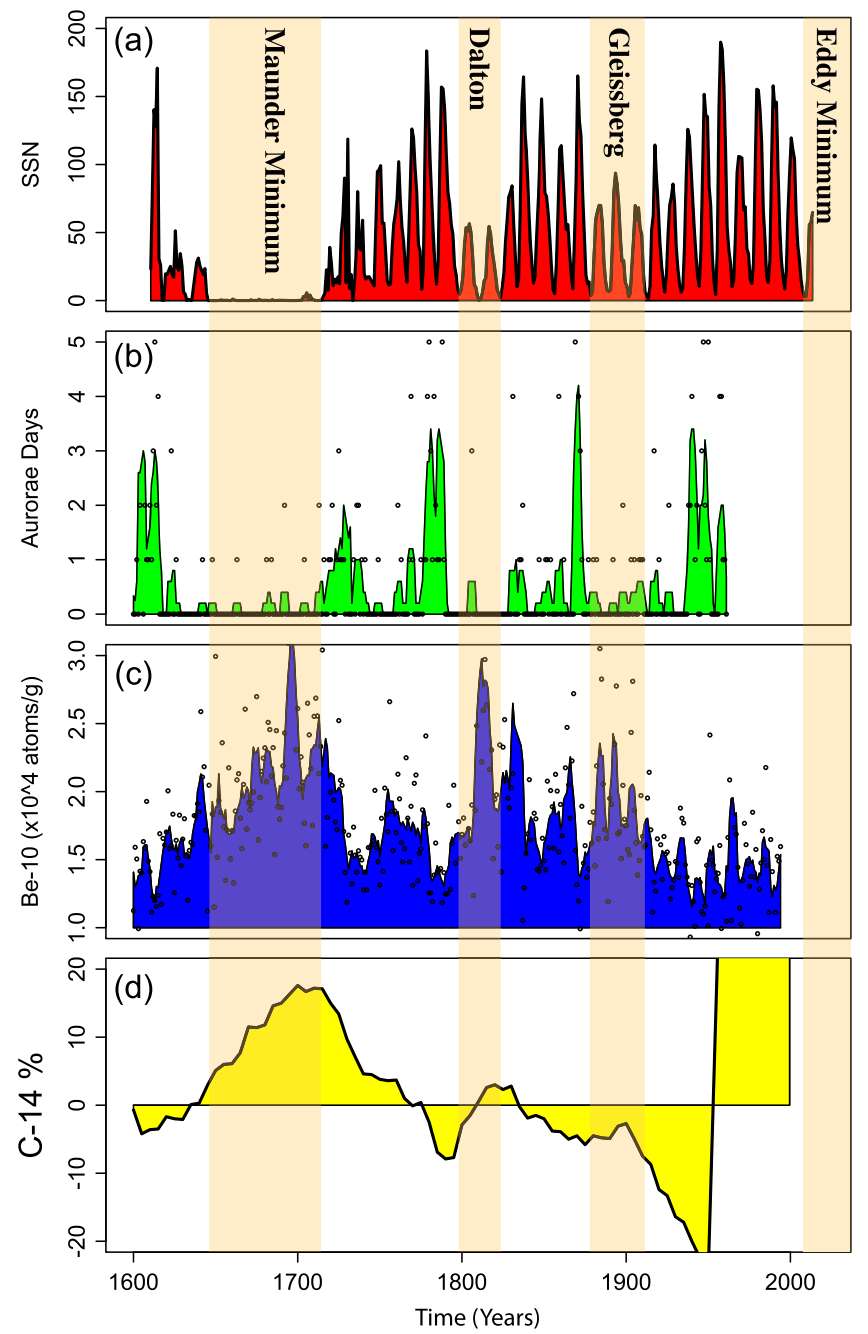

Figure 1. The evolution of various solar-related parameters from 1600 through 2012: (a) the yearly sunspot number (Lockwood et al. 2014a); (b) the number of aurora per year (Réthly \& Berkes 1963, p. 1523); (c) beryllium-10 measurements (Berggren et al. 2009); and (d) carbon-14 measurements (Reimer et al. 2004). The circles in (b) and (c) represent the original data at yearly resolution. See text for more details.

Assuming that the SSN record is a reliable proxy for the number of sunspots, we infer that in $\approx 1645$ they suddenly, and almost completely "turned off," and remained so until $\sim 1700-1715$, depending on how you interpret the extremely small cycle starting shortly after 1700 . Thus, from the perspective of the SSN, the Maunder interval was a period of constant inactivity. It is worth noting here that the point in the solar cycle at which the Maunder Minimum began could have important theoretical ramifications. Mackay (2003) argued that if the Maunder Minimum began at solar maximum, this would have led to a configuration with effectively no unipolar polar fields. In contrast, if it began near, or at solar minimum, strong unipolar polar fields would have been present that may have remained intact to a large extent throughout the interval.

At the risk of over-interpreting the signal, the SSN record during the Maunder Minimum further suggests that sunspots did cluster into perhaps half-a-dozen or so small peaks that may be suggestive of a continued solar cycle within the Maunder Minimum. Additionally, these cluster peaks, and the underlying base, tended to decrease slightly, perhaps suggesting that the Sun sank to even lower states of inactivity during the $70 \mathrm{yr}$ period. Ribes \& Nesme-Ribes (1993) analyzed measurements made at the Paris Observatory and concluded that: (1) the solar cycle continued to operate during the Maunder Minimum; and (2) the majority of sunspots appeared in the southern hemisphere, clustered around $\sim 10^{\circ} \mathrm{S}$.

\subsection{Aurora Observations}

Records of aurorae date back thousands of years (Siscoe 1980), but, again, come with caveats. Does the absence of an aurora mean that one did not occur, or just that it was not reported? Additionally, different records provide, at best, only threshold indicators of geomagnetic activity. Consistent observations at mid-latitudes, for example, will not contain modest geomagnetic events that reveal themselves only at high geomagnetic latitudes. Perhaps the strongest statement we can make is that if an aurora was observed, then it probably happened (allowing for confusion with other atmospheric phenomena), whereas, if none were reported, it may or may not mean that none occurred. Thus, the auroral record, at least older than a century, at best provides a lower limit of geomagnetic activity.

In spite of these limitations, several records exist and have been analyzed in detail. Here, for illustration, we rely on the dataset reconstructed by Réthly \& Berkes (1963), however, our conclusions would not be affected had we used another set of measurements (e.g., Schröder 1992; Silverman 1992). We consider the raw counts and smoothed profile shown in Figure 1(b) to make the following points. First, geomagnetic activity did not cease during the Maunder Minimum period. Second, activity was higher both before and after the Maunder Minimum. Third, there is a tentative suggestion of a solar cycle modulation in the number of aurora days.

The presence of any aurora indicates that the Sun, through the solar wind, was connected to the Earth's upper atmosphere. To generate aurora at middle and low latitudes requires a dawn-dusk electric field, which in turn, requires a roughly radially directed solar wind carrying a $B_{z}$ magnetic field. Additionally, the energy to accelerate ions and electrons toward Earth and generate aurora by bombarding oxygen and nitrogen atoms is supplied from the kinetic energy density of the solar wind flow (Cowley 1991). We conclude then, that Parker's suggestion that there might not be any solar wind at all, is not consistent with these observations.

Since these observations were made at mid-European latitudes, we can also infer that they were probably related to relatively substantial geomagnetic storms. This, in turn, suggests that they originated not from corotating interaction regions, which would tend to produce minor events, but from coronal mass ejections (CMEs). Further, we could anticipate that these CMEs were associated with the appearance of the relatively few sunspots that were present, signaling the presence of active regions. It is from active regions that the strongest (i.e., fastest and largest field strengths) CMEs are typically produced. Unfortunately, the limited number of auroral sightings during the Maunder Minimum (one at most (at all latitudes) in any one year, except once when two were observed) does not allow us to make a meaningful correlation between sunspot number and number of aurorae. In contrast, later in the record, there is a significantly clearer correlation between the temporal location of the peaks in the two time series (although no obvious association in their amplitude). 


\subsection{Cosmic Ray Fluxes Inferred from Cosmogenic Records}

Cosmogenic radionuclides are rare isotopes created when a high-energy cosmic ray interacts with the nucleus of an atom in the Earth's atmosphere. The two principal types used in longterm solar studies are ${ }^{10} \mathrm{Be}$ and ${ }^{14} \mathrm{C}$. Here, we focus principally on the former, since it is more directly relatable to cosmic ray intensities (Steinhilber et al. 2012). High-energy galactic cosmic ray particles impact primarily atmospheric nitrogen or oxygen, producing ${ }^{10} \mathrm{Be}$, which then becomes attached to aerosols. Depending on where the ${ }^{10} \mathrm{Be}$ is produced it may take from weeks (troposphere) to a year or two (stratosphere) before being deposited in the polar ice caps. Since climate/ precipitation effects can modulate the ${ }^{10} \mathrm{Be}$ concentrations within the ice, care must be taken when interpreting the records as a measure of cosmic ray intensities. The general consensus, however, is that ${ }^{10} \mathrm{Be}$ records provide, primarily, a measure of production rates (Usoskin 2008).

In panel (c) of Figure 1 we show the ${ }^{10} \mathrm{Be}$ record as reported by Berggren et al. (2009). The individual circles are yearly measurements of concentration. The black/blue curve represents an $11 \mathrm{yr}$ running average. We note several points. First, the solar cycle is clearly seen, particularly after 1895 (the beginning of the "Gleissberg" minimum). Second, the absolute concentration varies by a factor of $\sim 2.5$ over the $400 \mathrm{yr}$ interval. Third, the three established minima (Maunder, Dalton, and Gleissberg) all coincide with local peaks in concentration. Fourth, a striking feature is that, unlike the sunspot record, the Maunder Minimum as viewed through ${ }^{10} \mathrm{Be}$ was not steady. The ${ }^{10} \mathrm{Be}$ data steadily increased from $1.75 \times 10^{4}$ atoms/g to over $3 \times 10^{4}$ during the $70 \mathrm{yr}$ interval. Thus, at least from a cosmic ray perspective, the Maunder Minimum period was a period of evolution, not constancy.

If we assume that the ${ }^{10} \mathrm{Be}$ fluctuations are a reasonable fiduciary for variations in CR flux, we might infer that the CR flux increased by almost a factor of two, suggestng a commensurate decrease in the strength of the interplanetary magnetic field. In fact, the relationship between CR flux and the strength of the IMF is considerably more complicated (Usoskin 2008). We will return to this point in Section 2.5, where we consider the modulation potential.

The ${ }^{10} \mathrm{Be}$ record also calls into question the idea that the Maunder Minimum interval is substantially the same as the $2008 / 2009$ minimum. In particular, the recent minimum is a snapshot of the Sun in time, whereas, as we have argued here, the Maunder Minimum was an evolving configuration: If the recent minimum is related to the Maunder Minimum, which part is it associated with? Presumably, it would be the beginning, since we have not yet witnessed the $70 \mathrm{yrs}$ of little-to-no sunspots or the continual increase in cosmic ray fluxes. We conclude then, that at best, the 2008/2009 minimum may turn out to be the first minimum that defined the start of a descent toward a new grand minimum.

Finally, in panel (d) of Figure 1 we show yearly averages of the ${ }^{14} \mathrm{C}$ record as reported by Reimer et al. (2004). Again, a large value of ${ }^{14} \mathrm{C}$ suggests a stronger flux of cosmic rays, which in turn suggests a lower interplanetary field, possibly in conjunction with a relatively flat HCS. We note the almost monotonic increase from 1600 until shortly after 1700, consistent with the ${ }^{10} \mathrm{Be}$ record. Between then and 1955, it appears to generally decrease, except for two "recoveries" when it rises. The first occurred in $\sim 1800$ and the second, shortly before $\sim 1900$, coinciding with the Dalton and
Gleissberg minima. Above-ground nuclear tests began in 1955 rendering the time series from this point forward useless for present purposes. In fact, secular variations after $\approx 1850-1900$ are probably dominated by the anthropogenic effects of fossil fuel burning (Suess 1955).

Comparing the ${ }^{14} \mathrm{C}$ and ${ }^{10} \mathrm{Be}$ profiles, we note that, on the largest scales, they convey a similar trend for the inferred flux of cosmic rays. The three grand minima (seen as maxima in these records) are approximately co-temporal and the general variations about some reference point, say 1600, are reasonably matched. One notable exception is that while the peak ${ }^{10} \mathrm{Be}$ values during the Maunder and Dalton intervals match one another, they are substantially different in the ${ }^{14} \mathrm{C}$ record, perhaps the result of being superimposed on a monotonic decrease from 1700 until at least 1955.

From this, we conclude that the cosmic ray flux was larger during the Maunder Minimum than at any time over the last 400 yrs. Moreover, the flux of particles systematically increased during the $70 \mathrm{yr}$ interval, suggesting that the minimum was "flat" only from the perspective of sunspots. In summary then, the Maunder Minimum did not reach its most profound state until 1698, 43 yrs after the "nominal" onset, as defined by the sunspot record.

\subsection{Eclipse Observations}

Observations of eclipses during the Maunder Minimum are strongly suggestive, but not conclusive, that the structured corona observed in modern times during eclipses disappeared (Eddy 1976). Here, we build upon, and add to the evidence compiled by J. Eddy. As he noted, of the 63 possible solar eclipses known to have occurred between 1645 and 1715, only eight passed through Europe, and, of those, four (1652, 1698, 1706 , and 1708) were captured by reports sufficiently detailed to be of use for this study.

Dr. John Whybard gave an account of his, and that of the vice-prefect's observations of the 1652 solar eclipse in Carrickfergus, located in County Antrim, Northern Ireland (Wing 1656). He stated that the corona "had a uniform breadth of half a digit, or a third of a digit at least, that it emitted a bright and radiating light, and that it appeared concentric with the Sun and moon when the two bodies were in conjunction."

As reported by Grant (Grant 1852), M. M. Plantade and Capies observed the eclipse of 1706 at Montpellier, located on the south coast of France, in a way that was "clearer and more precise than any other that had been hitherto recorded." They observed that "as soon as the Sun was totally eclipsed, there appeared around the moon a very white light forming a kind of corona, the breadth of which was equal to about $3^{\prime}$. Within these limits the light was everywhere equally vivid, but beyond the exterior contour, it was less intense, and was seen to fade off gradually into the surrounding darkness, forming an annulus around the moon of about 8 degrees diameter."

Both sets of reports describe a structureless corona composed of a ring of light circumscribing the entire moon. Had the Sun displayed a dipolar or quadrupolar configuration, such as any of the eclipses viewed in, say, the last century, we might have expected the observers to note this fact.

The eclipse of 1715, which was well observed from London, however, lays some doubt on this inference. Halley himself reported that there "appeared a luminous ring around the moon as on the occasion of the eclipse of 1706," suggesting at least a qualitative similarity between the two events. R. Cotes, on the 
other hand, wrote "besides this ring, there appeared also rays of a much fainter light in the form of a rectangular cross...The longer and brighter branch of this cross lay very nearly along the ecliptic, the light of the shorter was so weak that I did not constantly see it." Eddy (1976) interpreted the longer, brighter branch to be a description of a solar minimum streamer belt configuration and the shorter branch to be polar plumes. Additionally, observations of a "red flash" during the 1706 and 1715 eclipses suggest the presence of a significant photospheric magnetic field (Foukal \& Eddy 2007). The discrepancy between these two accounts raises several issues. First, it is possible that earlier accounts failed to acknowledge that there was an underlying structure. Or, second, that the structured Kcorona had returned to the Sun by the time of the 1715 eclipse.

The eclipse of 1766, which was observed "in the Southern Ocean by the persons on board the French ship of war the Comte d'Artois," provides clear evidence that "normal" solar conditions had returned. Although totality only lasted some 53 seconds, the observers noted "a luminous ring about the moon, which had four remarkable expansions situate at a distance of 90 [degrees] from each other." From this we can infer that a quadrupolar streamer structure was visible.

Finally, we remark that during the eclipses of 1652, 1698, 1706, and 1708, the corona was described as "dull or mournful," and often as "reddish," which Eddy (1976) suggested might describe how the zodiacal light component (i.e., the F-corona) might look to an observer in the absence of a K-corona.

In summary then, we conclude that: (1) the corona during the Maunder Minimum was likely featureless, at least to the extent that it was not commented on; (2) the coronal light that was present was "reddish" and (3) coronal features likely returned sometime between 1708 and 1766 .

\subsection{Cosmic Ray Modulation Potential}

Variations in cosmogenic radionuclide records, and, in particular ${ }^{10} \mathrm{Be}$ and ${ }^{14} \mathrm{C}$, are believed to provide a measure of solar activity (Usoskin 2008). To a rough approximation, their values indicate the flux of cosmic rays impinging the upper atmosphere. However, the transport and deposition mechanisms for both species are relatively complex and quite different from one another, meaning that interpretation does not come without important caveats.

A number of both empirical and physics-based models have been developed to recover unbiased estimates of "solar activity." Here, we focus on the so-called modulation potential, $\phi$. As suggested by its name, $\phi$ is intended to capture the variability in the observed cosmic ray flux in the vicinity of Earth. Because these fluxes are modulated on a global scale, $\phi$ is a global heliospheric quantity, capturing the physical processes of: (1) diffusion of particles due to scattering; (2) convection in the solar wind; and (3) adiabatic losses. The following empirical estimate for $\phi$ provides an intuitive way to understand it:

$$
\phi=\phi_{o}+\phi_{1}\left(\frac{F}{F_{o}}\right)^{1+\frac{\alpha}{\alpha_{o}}}(1+\beta p)
$$

where $F$ is the OSF, $\alpha$ is the tilt angle of the HCS, and $p$ is the global magnetic polarity; $p=1(-1)$ for positive (negative) polarity periods. Best fit values for the constants are:

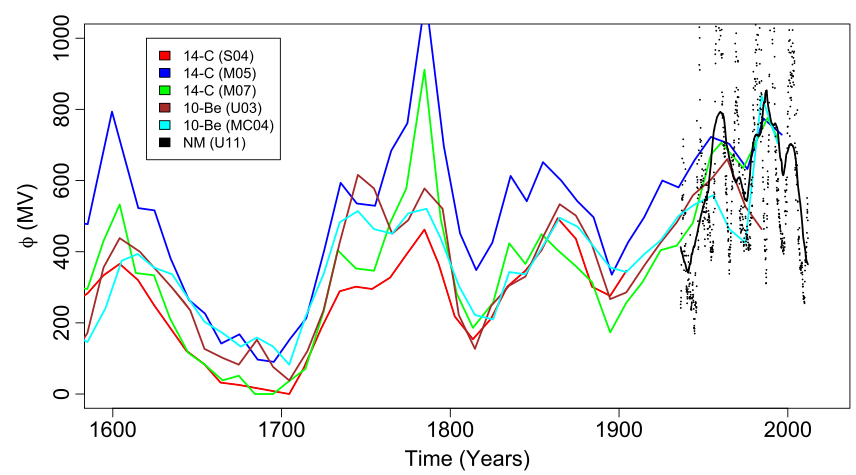

Figure 2. Temporal evolution of a selection of estimates for the modulation potential $(\phi)$. Following Usoskin (2008), S04 refers to Solanki et al. (2004), M05 refers to McCracken et al. (2005), M07 refers to McCracken \& Beer (2007), U03 refers to Usoskin et al. (2003), MC04 refers to McCracken et al. (2004), and U11 refers to Usoskin et al. (2011). The first three profiles are based on carbon-14 records, the next two are based on beryllium-10, and the final profile shows direct neutron monitor and ground-based ionization chamber measurements both on monthly averages (dots) and an $11 \mathrm{yr}$ running mean (solid black curve).

$\phi_{o}=150 \mathrm{MV}, \phi_{1}=86 \mathrm{MV}, F_{o}=2.5 \times 10^{14} \mathrm{~Wb}, \alpha_{o}=91^{\circ}$, and $\beta=-0.03$ (Alanko-Huotari et al. 2006).

From Equation (1), we can appreciate that during solar minimum periods, when $\alpha \rightarrow 0^{\circ}$, the modulation potential is linearly proportional to the open flux in the heliosphere. During elevated periods of activity (and in the extreme that $\alpha \rightarrow 90^{\circ}$ ) the modulation potential is more sensitive (up to the square) of the open flux. Intuitively, this makes sense: during periods of higher activity, as the heliospheric magnetic field strength increases and the latitudinal extent of the HCS broadens there is a larger barrier for cosmic rays to impact the Earth. In contrast, when the HCS becomes flat, and the field strength reduces, the structure of the solar wind provides less of an impediment to the propagation of these particles. Of the two parameters, since $\alpha$ varies between $10^{\circ}$ and $90^{\circ}$ every 11 yrs, it does not impact $\phi$ as much as $F$, which varies by more than a factor of two. Moreover, $\alpha$ must oscillate between these extremes every cycle, and cannot drift beyond them on longer times scales as can $F$. Therefore, we conclude that long-term variations in $\phi$ likely represent changes in the large-scale heliospheric magnetic field strength.

Armed with this simplified picture, we can now interpret several reconstructions of the modulation potential shown in Figure 2. The solid colored lines show various estimates of $\phi$ using both ${ }^{10} \mathrm{Be}$ and ${ }^{14} \mathrm{C}$ records. The black solid line is an $11 \mathrm{yr}$ running mean of monthly averages (black points) of $\phi$ derived from neutron monitor and ground-based ionization chamber measurements. The approximate match between the cosmogenic records and the neutron monitor measurements gives us some confidence that present day values can be-at least roughly - compared with historical estimates, particularly during the deepest portion of the Maunder Minimum $(\approx 1700)$. However, more important than the absolute values is the variability in the cosmogenic data between 1600 and 2000. If we assume that, to first order, $\phi$ is providing a proxy for the strength of the heliospheric magnetic field, then its strength during the Maunder Minimum was as much as 500/50 $=10$ times lower than it was during the decade beginning in 2000. Additionally, the field strength during the early 1600s, which, it could be argued, was the beginning of a long term, monotonic decrease in field strength, is approximately the same as the 
inferred field strength today, reinforcing the suggestion by Barnard et al. (2011) that we may be entering a grand solar minimum, similar to the Maunder interval.

These profiles also promote the idea that the Maunder Minimum was not an extended interval of constant inactivity, as might be inferred from the sunspot record, but a progressive drop, culminating in a deep minimum. It is worth noting, however, that while this minimum could indicate that the Sun had reached some "floor" level of activity, this is not necessarily the case. The decay implied by the ${ }^{10} \mathrm{Be}$ data might have continued had solar activity, and the magnetic flux emergence associated with it, not recovered. That recovery itself may have been triggered by a certain level of activity being reached or it might have resulted from independent processes, in which case, even 1700 does not set a base level for solar activity. It should be noted, however, that Steinhilber et al. (2012), who estimated $\phi$ from the common cosmic ray intensity record, showed that values during the Maunder Minimum were the lowest reached during any time within the last 10,000 yrs.

\section{CANDIDATE SCENARIOS FOR THE SUN'S PHOTOSPHERIC MAGNETIC FIELD DURING THE MAUNDER MINIMUM}

The Sun's photosphere provides a convenient boundary from which to base our calculations. First, the photospheric magnetic field is well observed by both ground-based and space-based solar observatories. Second, the transition from a flowdominated to a magnetic field-dominated environment occurs at the photosphere. In principle then, assuming that all of the salient physical processes are included in the models, and that the model results are not strongly dependent on the values of free parameters (i.e., coefficients in the formulation of the relevant physics that are not well constrained), specification of the photospheric magnetic field should be sufficient to reconstruct the global structure of the corona and inner heliosphere. Such models are frequently applied to data from the modern era driven by the observed photospheric magnetic field, in an effort to understand the large-scale structure of the corona and inner heliosphere, and generally match the observed large-scale structure of the inner heliosphere (e.g., Riley et al. 2011, 2012).

The distribution of magnetic field in the photosphere during the Maunder Minimum, however, is subject to considerable speculation. We have reasonably reliable evidence that few or no sunspots were observed during this period, suggesting the absence of active regions. Theoretically, several studies have speculated on various aspects of the Maunder Minimum solar field. Schrijver et al. (2011) argued that small-scale fields associated with ephemeral regions must have persisted during even the deepest portion of the interval. Mackay (2003) proposed that the Maunder Minimum must have commenced at, or near solar minimum: had it started at solar maximum, there would have been no reversal of the polar fields, in apparent conflict with evidence that the solar cycle continued to operate during this $70 \mathrm{yr}$ period (e.g., Beer et al. 1998; Cliver et al. 1998; Usoskin et al. 2001; Miyahara et al. 2004; Berggren et al. 2009). It is also worth noting, however, that it is possible that the cyclic behavior during the Maunder Minimum was not a reduced-amplitude version of what is seen in more active intervals. Owens et al. (2012) have argued that the cycles originate from variations in the loss rate of OSF rather than the variations in production rate, which dominate in more active periods. In addition, Usoskin et al. (2014) presented some evidence that during the Maunder Minimum the Sun was in an entirely different mode of variation. Thus, one approach to deducing the photospheric field during this interval is to construct a range of possible scenarios and test their predictions against the available, albeit limited observations.

From the discussion in Section 2.5, it is reasonable to discount the extreme possibility, raised (but not necessarily advocated) by Parker (1976), that the entire visible magnetic field disappeared. While this would provide an obvious means for removing all sunspots, we know: (1) that there were very occasional sunspots during the Maunder interval; (2) there were occasional geomagnetic storms; and (3) at least the suggestion of some form of a solar cycle still operating during this interval. The continued, sporadic appearance of sunspots suggests that magnetic flux continued to emerge through the photosphere, albeit at a much lower rate. The occasional geomagnetic storms suggest a continued magnetic connection between the solar surface and Earth's magnetosphere. And the maintenance of a solar cycle (albeit potentially a rather different one) suggest that the field did not "extinguish" itself, which, from a theoretical perspective, would be difficult to envisage.

Thus, we suggest that the most radical scenarios for the Maunder Minimum photosphere may have contained only small-scale ephemeral flux, random in amplitude and position, but substantially less (say, one third to an order of magnitude) than currently observed ephemeral regions. Figures 3(e) and (f) summarize a photosphere composed of only parasitic polarity (i.e., small-scale ephemeral regions) of strength \pm 10 and $\pm 3.3 \mathrm{G}$, respectively. Strong field magnetic elements in the photosphere are typically $\sim 1 \mathrm{kG}$; however, flux densities are dependent on resolution. The value of $\pm 10 \mathrm{G}$ was chosen to match observed values from synoptic magnetograms assembled at this resolution $\left(1^{\circ} \times 1^{\circ}\right)$ during the 2008 time period, while the $\pm 3 \mathrm{G}$ value is simply a $1 / 3$ scaling. In these scenarios there are no large-scale polar fields, i.e., the dynamo as we know it would have ceased to operate. Evolutionarily, we might anticipate that this state was arrived at slowly as the polar fields decayed, not being replaced by poleward-migrating flux from sunspots, which had disappeared. Thus, this might represent the state of the Sun late in the Maunder Minimum interval.

At the other end of the spectrum, the most conservative scenario would be that the Maunder Minimum period was no different than the recent minimum of 2008/2009 (Figure 3(a)). If substantiated, this is an appealing result because all of the modern era measurements, modeling, and inferences could be applied to better understand, and constrain the Maunder interval. However, the fact that the 2008/9 minimum follows a strong cycle (\# 23) and the subsequent emergence of cycle 24 (albeit a relatively weak cycle) indicates that the 2008/9 minimum is a very limited analog for the state of the Sun for much of the Maunder Minimum.

To these scenarios, we add several alternatives. In the first (Figure 3(b)), we superimpose an axial dipole of strength $3.3 \mathrm{G}$ on top of a parasitic polarity distribution with peak amplitude of $\pm 30 \mathrm{G}$. In the second (Figure $3(\mathrm{c})$ ), we consider a dipole only, with strength $3.3 \mathrm{G}$. The third scenario reproduces case (b), except that the large-scale dipole is reduced to $1 \mathrm{G}$. 


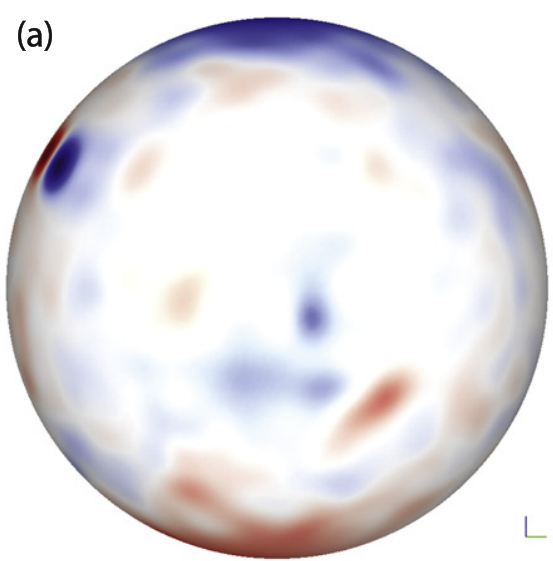

(b)

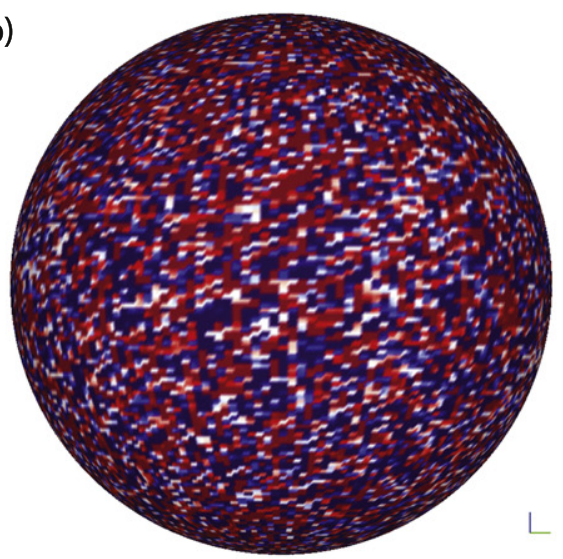

(d)

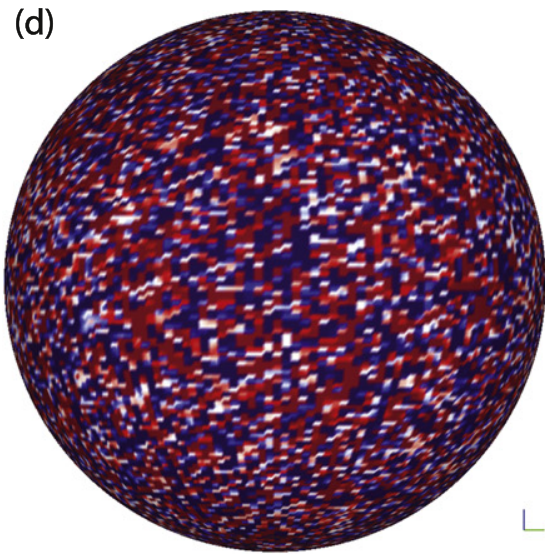

(e)

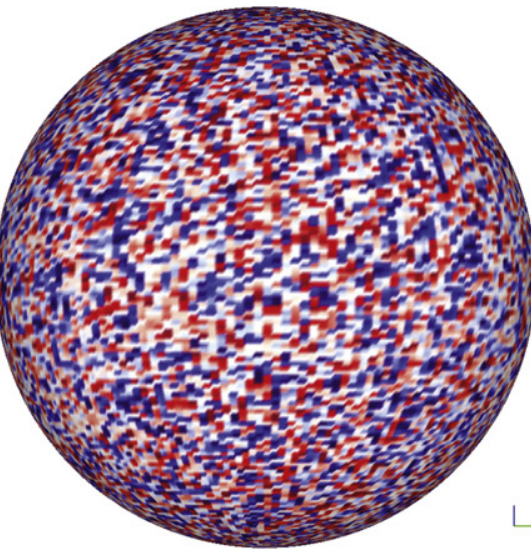

(c)

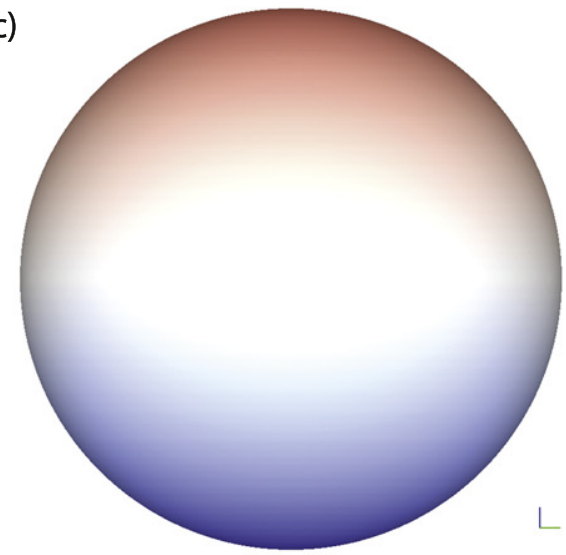

(f)

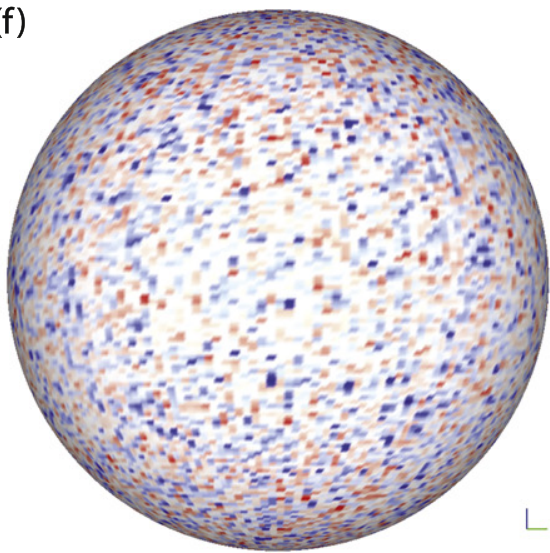

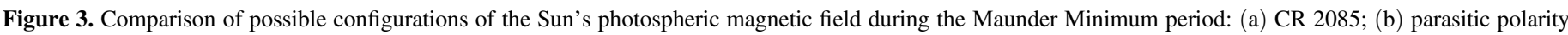

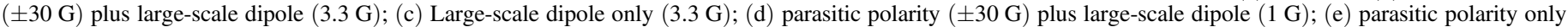
$( \pm 10 \mathrm{G})$; and (f) parasitic polarity $( \pm 3.3 \mathrm{G})$.

These six scenarios represent a wide possible array of configurations for the distribution of flux in the photosphere that may have existed during the Maunder Minimum.

\section{GLOBAL MHD MODELING}

A global MHD model of the solar corona and inner heliosphere can provide a unique and powerful way to "selfconsistently" link the disparate observations discussed in Section 2 and assess the likelihood that any of the scenarios discussed in Section 3 are consistent or in conflict with these observations. The model we describe in the following sections contains the key elements that: (1) the primary driver is the photospheric magnetic field; and (2) the heating of the corona is a function only of the photospheric magnetic field strength. Thus, the magnetic and emission properties of the corona are coupled, and we can, at least in principle, apply the model to epochs with significantly different properties.

\subsection{Model Description}

The MHD approximation is appropriate for large-scale, lowfrequency phenomena in magnetized plasmas such as the solar corona. Using the photospheric magnetic field as the primary driving boundary condition, as described in Section 3, we can attempt to reproduce the Sun's magnetic and emission properties during during the Maunder Minimum by solving the following set of viscous and resistive MHD equations:

$$
\begin{gathered}
\boldsymbol{\nabla} \times \boldsymbol{B}=\frac{4 \pi}{c} \boldsymbol{J}, \\
\boldsymbol{\nabla} \times \boldsymbol{E}=-\frac{1}{c} \frac{\partial \boldsymbol{B}}{\partial t}, \\
\boldsymbol{E}+\frac{\boldsymbol{v} \times \boldsymbol{B}}{c}=\eta \boldsymbol{J}, \\
\frac{\partial \rho}{\partial t}+\boldsymbol{\nabla} \cdot(\rho \boldsymbol{v})=0, \\
\frac{1}{\gamma-1}\left(\frac{\partial T}{\partial t}+\boldsymbol{v} \cdot \boldsymbol{\nabla} T\right)=-T \boldsymbol{\nabla} \cdot \boldsymbol{v}+\frac{m}{2 k \rho} S \\
\rho\left(\frac{\partial \boldsymbol{v}}{\partial t}+\boldsymbol{v} \cdot \nabla \boldsymbol{v}\right)=\frac{1}{c} \boldsymbol{J} \times \boldsymbol{B}-\nabla\left(p+p_{w}\right) \\
+\rho \boldsymbol{g}+\boldsymbol{\nabla} \cdot(\nu \rho \boldsymbol{\nabla} \boldsymbol{v}), \\
S=\left(-\boldsymbol{\nabla} \cdot \boldsymbol{q}-n_{e} n_{p} Q(T)+H_{\mathrm{ch}}\right),
\end{gathered}
$$

where $\boldsymbol{B}$ is the magnetic field, $\boldsymbol{J}$ is the electric current density, $\boldsymbol{E}$ is the electric field, $\rho, \boldsymbol{v}, p$, and $T$ are the plasma mass density, velocity, pressure, and temperature, $g=-g_{0} R_{\odot}^{2} \hat{\boldsymbol{r}} / r^{2}$ is the gravitational acceleration, $\eta$ the resistivity, and $\nu$ is the kinematic viscosity. Equation (8) contains the radiation loss function $Q(T)$ as in Athay (1986), $n_{e}$ and $n_{p}$ are the electron and proton number densities (which are equal for a hydrogen 
plasma), $\gamma=5 / 3$ is the polytropic index, $H_{\mathrm{ch}}$ is the coronal heating term (see below), and $\boldsymbol{q}$ is the heat flux.

For the present study, we have used a grid of $151 \times 180 \times 360$ points in $r \times \theta \times \phi$. The grid resolution is nonuniform in $r$ with the smallest radial grid interval at $r=R_{\odot}$ being $\sim 0.33 \mathrm{~km}$. The angular resolution in $\theta$ and $\phi$ is $1^{\circ}$. A uniform resistivity $\eta$ was used, corresponding to a resistive diffusion time $\tau_{R} \sim 4 \times 10^{3} \mathrm{hr}$, which is much lower than the value in the solar corona. This is necessary to dissipate structures that cannot be resolved which are smaller than the cell size. The Alfvén travel time at the base of the corona $\left(\tau_{A}=R_{\odot} / V_{A}\right)$ for $|\boldsymbol{B}|=2.205 \mathrm{G}$ and $n_{0}=10^{8} \mathrm{~cm}^{-3}$, which are typical reference values, is 24 minutes, and so the Lundquist number $\tau_{R} / \tau_{A} \approx 1 \times 10^{4}$. A uniform viscosity $\nu$ is also used, corresponding to a viscous diffusion time $\tau_{\nu}$ such that $\tau_{\nu} / \tau_{A}=500$. Again, this value is chosen to dissipate unresolved scales without substantially affecting the global solution. Our model starts at the upper chromosphere and includes the transition region (Lionello et al. 2009).

\subsection{Coronal Heating}

Global MHD models of the solar corona and inner heliosphere have demonstrated their ability to reproduce the essential features of a range of measurements and observations during the space era (e.g., Riley et al. 2011, 2012). A crucial aspect and limitation of current capabilities concerns the physical mechanisms that heat the corona. While it is generally believed that it must involve the conversion of magnetic energy into heat, it is not clear how this transformation takes place. One scenario involves the dissipation of high-frequency waves, while another relies on the rapid release of energy built up from slow photospheric motions (e.g., Lionello et al. 2009). Phenomenologically, it is well known that magnetic flux and X-ray radiance are linearly correlated over many orders of magnitude (Fisher et al. 1998; Pevtsov et al. 2003; Riley et al. 2010).

In this study, we take the pragmatic but necessary position of specifying the heating as a function of magnetic field strength. Specifically, we assume that the heating of the corona takes the following form:

$$
\begin{gathered}
H=H_{\mathrm{QS}}+H_{\mathrm{AR}} \\
H_{\mathrm{QS}}=H_{\mathrm{QS}}^{0} f(r) \frac{B_{t}^{2}}{B\left(\left|B_{r}\right|+B_{r}^{c}\right)} \\
H_{\mathrm{AR}}=H_{\mathrm{AR}}^{0} g(B)\left(\frac{B}{B_{0}}\right)^{1.2}
\end{gathered}
$$

where: $\quad B_{t}=\sqrt{B_{\theta}^{2}+B_{\phi}^{2}}, \quad H_{\mathrm{QS}}^{0}=1.18 \times 10^{-5} \mathrm{erg} \mathrm{cm}^{-3} \mathrm{~s}$, $B_{r}^{c}=0.55 \mathrm{G}, H_{\mathrm{AR}}^{0}=1.87 \times 10^{-5} \mathrm{erg} \mathrm{cm}^{-3} \mathrm{~s}$, and $B_{0}=1 \mathrm{G}$, $f(r)=\exp \left(-\frac{r / R_{\odot}-1}{0.2}\right)$, and $g(B)=\frac{1}{2}\left(1+\tanh \frac{B-18.1}{3.97}\right)$.

Although these functions are ad hoc, importantly, they depend only on the strength of the magnetic field, and hence, provided that coronal heating too depends only on field strength, should be applicable to a range of values even outside those that have been observed during the space era. Thus, by demonstrating that the model can reproduce the basic features of the observations during the space era, and requiring that the heating profiles depend only on the magnetic field, that is, that there are no additional free parameters, we have some confidence that the heating profiles should be applicable when applied to more extreme conditions.

\subsection{Computation of the Open Solar Flux}

The OSF is that portion of the Sun's magnetic field that enters into the heliosphere (Lockwood 2013). Assuming further that, on sufficiently long temporal scales, this flux is independent of position in the heliosphere, as suggested by Ulysses observations (Smith \& Marsden 2003), in situ measurements of the interplanetary magnetic field, $B_{\mathrm{IMF}}$, are a proxy for the open flux. Multi-solar cycle measurements of OSF demonstrate that the open flux roughly doubles between solar minimum and solar maximum. Owens \& Crooker (2006), Riley et al. (2007) and Schwadron et al. (2010) have argued that the measured flux at $1 \mathrm{AU}$ consists of a relatively constant background flux, with an additional contribution from CMEs, which at solar maximum can be as large as the background level.

Lockwood et al. (2009) have shown that OSF can be predicted from near-Earth measurements of $\left|B_{r}^{\mathrm{IMF}}\right|$ provided the radial IMF component $\mathrm{B}_{r}^{\mathrm{IMF}}$ is averaged over a day before the modulus is taken. This averaging time scale is a compromisebeing short enough to avoid large-scale canceling out of the genuine toward-and-away source structure yet long enough to average out smaller scale structure in the IMF. This averaging time constant was employed by Wang \& Sheeley (1995), and we can adopt their equation to estimate the magnitude of the radial IMF at Earth from the MHD solution as:

$$
\left|B_{r}^{E}\right|=\frac{\left|\Phi_{\text {open }}\right|}{4 \pi r_{E}^{2}}=\frac{1}{4 \pi}\left(\frac{R_{s}}{215 R_{\odot}}\right)^{2} \int\left|B_{r}\left(R_{s}, \theta, \phi\right)\right| d \Omega
$$

where $\phi$ denotes longitude, $R_{\odot}$ radius of the Sun, $R_{S}$ is the radius of the source surface, typically $2.5 R_{\odot}, r_{E}$ is the distance of the Earth from the Sun (1 AU), and the solid-angle integral is computed over a sphere at the source surface.

\subsection{Model Results}

We used the six magnetic maps described in Section 3 to compute MHD model solutions of the solar corona from 1 to $30 R_{S}$. The resulting magnetic field configurations are shown in Figure 4. The same starting points were used in each panel, corresponding to a mesh resolution of $10^{\circ}$ in latitude and longitude. We note several points. First, a relatively clear streamer belt configuration can be discerned for cases $(a)-(d)$ : field lines emanating from the polar regions extend into interplanetary space and remain open. Those straddling the heliospheric equator tend to be closed. Second, there is a qualitative decrease in the amount of open flux in moving from (a) to (f), at least based on the number of field lines drawn. Third, there is a net decrease in the organization of field lines from (a) to (f) (except for (c), which displays perfect axial symmetry). This is particularly true for comparisons between (a)-(d) and (e)-(f), the latter showing no obvious axis of symmetry. Interestingly, (d), although displaying axial symmetry, appears to be tilted substantially with respect to the rotation axis, presumably because of the presence of a coherent feature in the parasitic polarity.

For each solution, we computed the open magnetic flux, as defined by Equation (12). These are summarized in Table 1. Our "standard run," CR 2085, produced a radial IMF of 1nT at 

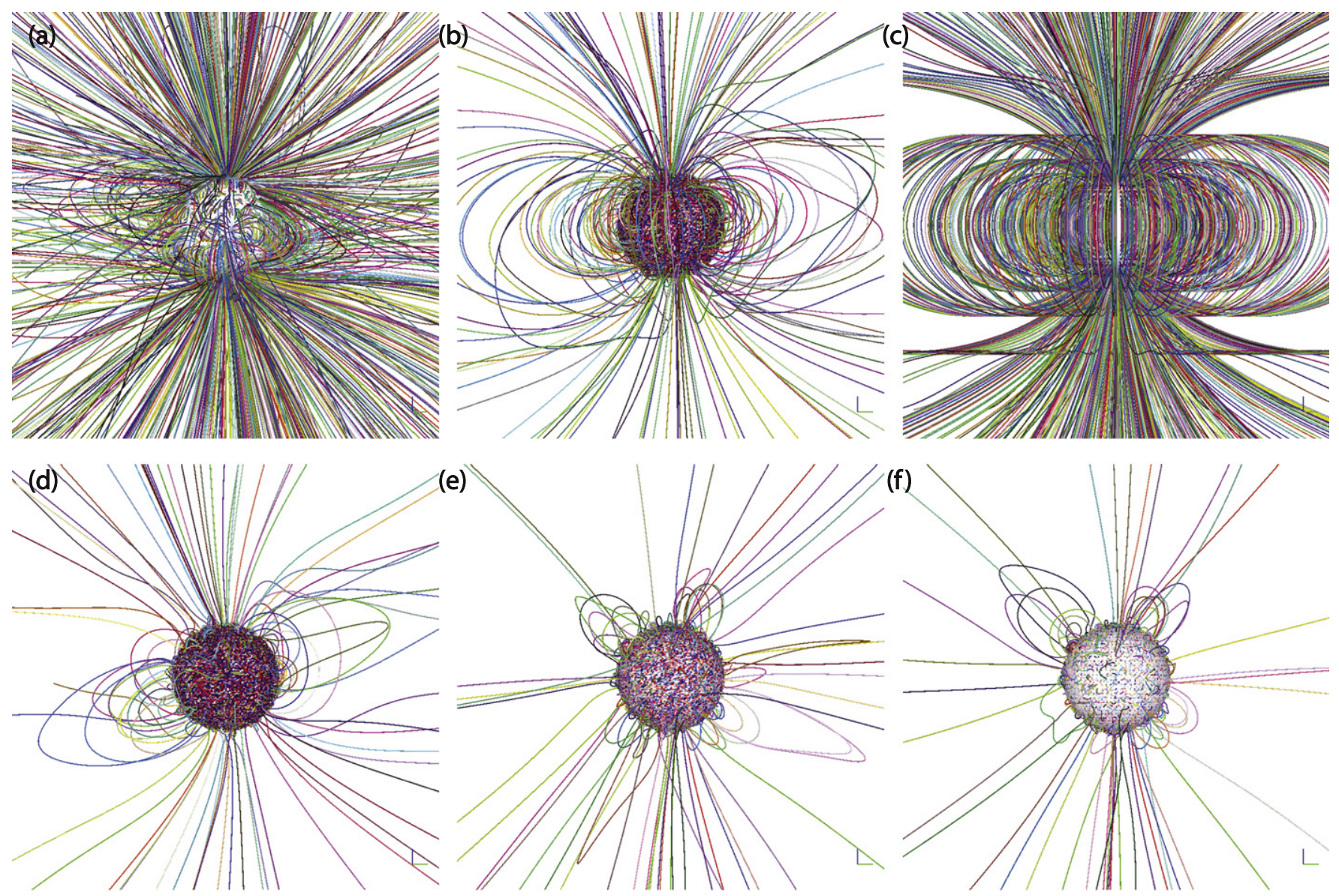

Figure 4. As Figure 3 but showing a selection of magnetic field lines drawn from a grid separated by $10^{\circ}$ in latitude and longitude.

Table 1

Open Flux Estimates

\begin{tabular}{lcc}
\hline \hline Model & Description & Open Flux \\
\hline (a) & CR $2085(06 / 26 / 09-07 / 23 / 09$, polar field $\sim 1.9 \mathrm{nT})$ & $1.0 \mathrm{nT}$ \\
(b) & Parasitic polarity $\left( \pm 30 \mathrm{G}^{\mathrm{a}}\right)+$ Large-scale & $2.4 \mathrm{nT}$ \\
& dipole $(3.3 \mathrm{G})$ & \\
(c) & Large-scale dipole only $(3.3 \mathrm{G})$ & $2.2 \mathrm{nT}$ \\
(d) & Parasitic polarity $( \pm 30 \mathrm{G})+$ Large-scale dipole $(1 \mathrm{G})$ & $1.2 \mathrm{nT}$ \\
(e) & Parasitic polarity only $( \pm 10 \mathrm{G})$ & $0.29 \mathrm{nT}$ \\
(f) & Parasitic polarity only $( \pm 3.3 \mathrm{G})$ & $0.08 \mathrm{nT}$ \\
\hline
\end{tabular}

${ }^{\mathrm{a}}$ Maximum amplitude.

1 AU. Scenario (b) results in the largest radial IMF $(2.4 \mathrm{nT})$, then scenarios (c) through (f) yield progressively less radial field. Radial fields of $1 \mathrm{nT}$ and $2.4 \mathrm{nT}$ correspond to OSFs of $2.8 \times 10^{14} \mathrm{~Wb}$ and $6.8 \times 10^{14} \mathrm{~Wb}$, respectively. By way of comparison, from modeling based on geomagnetic reconstructions of the OSF and continuity equations and using sunspot numbers to compute the CME and hence flux emergence, Lockwood \& Owens (2014) recently arrived at OSF estimates during the Maunder Minimum of between $0.8 \times 10^{14} \mathrm{~Wb}$ and $1.6 \times 10^{14} \mathrm{~Wb}$, the uncertainty being derived from Monte-Carlo analysis of the fitting, but also including the cyclic behavior during the Maunder Minimum. Note that their plot shows signed OSF and so values must be multiplied by two to compare with the unsigned fluxes presented here. Thus their values overlap with those derived here but are slightly lower on average.

The largest proportional change occurs for (e) to (d)-a factor of 4.1, followed by the change from (f) to (e) - a factor of 3.6. The value computed for CR 2085 (scenario (a)), is lower than estimates made using near-Earth spacecraft (and earlier observations from Ulysses). However, the relative variations in open flux between models and observations have been shown to match well (Wang \& Sheeley 1995). Stevens et al. (2012) have investigated the known deficit in the open flux produced by the models, suggesting that a better estimate of some of the model parameters (e.g., coronal base temperature) may resolve the difference. Additionally, more recent analysis by Linker et al. (2012) suggests that current synoptic maps may be underestimating the polar field strengths, which would lead to a systematic reduction in the model estimates for the open flux. For our purposes, assuming that the relative differences are reasonably accurate, scenarios (e) and (f) predict reductions over 2008 conditions of a factor of $\approx 3.4$ and $\approx 12.5$, respectively. Additionally, it should be noted that the 2008 time period we are comparing to represents a somewhat unique interval where the fields were lower by a factor of 1.6 over the previous space era minima (Smith \& Balogh 2008), and general solar activity was estimated to be the lowest it had been in the last century (e.g., Riley et al. 2011). The range bracketed by scenarios (e) and (f) is 

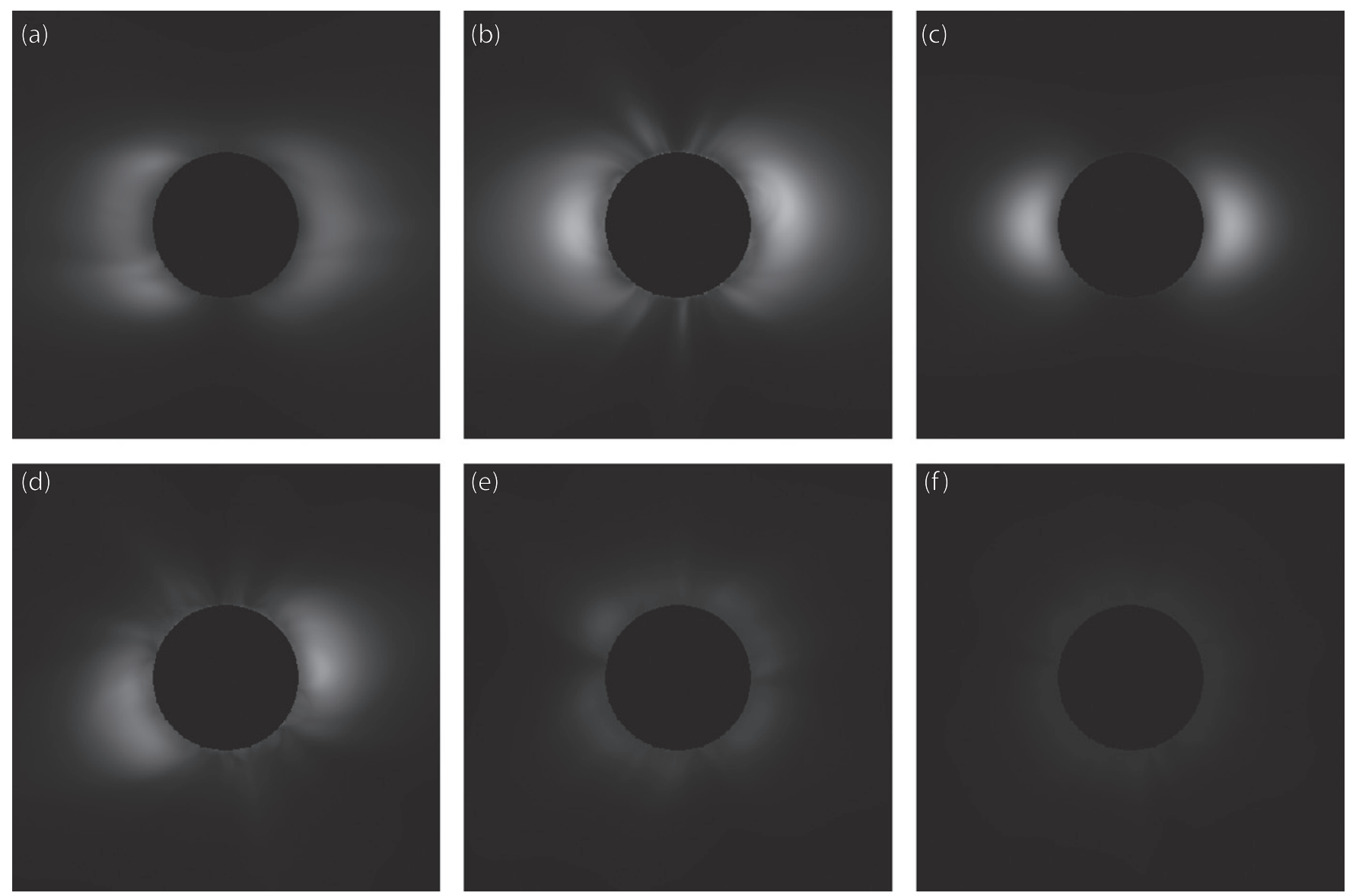

Figure 5. As Figure 3 but showing simulated polarized brightness.

consistent with the difference between the curves in Figure 2 when contemporary values are compared with those in 1700 .

We next consider the structure of the corona in white light that these scenarios suggest. In Figure 5, we have computed the simulated polarized brightness $(p B)$ for each solution. These were constructed by integrating the plasma along the line of sight with a suitable weighting function (Billings 1966). We have found that the model usually matches observed white light images both from spacecraft and ground-based observations during eclipses (Riley et al. 2001, 2011; Mikić et al. 2007; Riley 2010). Scenario (a), which represents the corona during the last solar minimum, displays the typical mid- and lowlatitude streamer structure we expect during the declining phases and solar minimum. The recent minimum was unique in that there were a larger number of pseudo-streamers present than during the previous (1996) minimum, which led to a broader and more structured "belt" of brightness around the equator (Cliver \& Ling 2011; Riley \& Luhmann 2012). The presence of unipolar polar fields is clearly seen by the dark regions over both poles. Scenario (b), which represents an idealization of scenario (a) by removing any large-scale active regions, as well as scenario (c) for which the parasitic polarity has been removed, present similar pictures. The closed, dipolar fields bracketing the equator trap plasma that scatters photons to the observer while the polar, open field regions retain only a tenuous outwardly streaming flow of plasma that cannot be easily seen in white light. Scenarios (e) and (f) are both much darker and do not display any axial symmetry. It is likely that scenario (f) would not be visible to the naked eye, especially if contrasted with the relative brightness of the preceding partial eclipse. Scenario (e) is marginally more visible, and appears to show some structure. Whether or not this would be reported, or whether only the more dominant effect of a "halo" or "annulus" would be noted by observers of the time is unclear. Scenario (d), which in some sense was designed to bridge the gap between (a)-(c) and (e)-(f) by reducing the dipole strength by a factor of 3.3, clearly shows a streamer-belt-like configuration. We conclude from these images that, had scenarios (a) through (d) been in effect during the Maunder Minimum, observers would probably have noted the existence of structure within the white-light corona.

The white light we observe from the solar corona is made up of two primary components: the $\mathrm{K}$ (kontinuierlich) and $\mathrm{F}$ (Fraunhofer) corona. The K-corona is created by sunlight scattering off free electrons, while the F-corona is created by sunlight scattering off dust particles. Close to the Sun, the Kcorona dominates; however, beyond $\approx 3 R_{S}$ (the precise number depending sensitively on the point of observations and solar conditions), the brightness of the F-corona exceeds that of the K-corona (Koutchmy \& Lamy 1985). Here, it is important to differentiate between brightness, $B$, and polarized brightness, $p B$ : while coronagraphs (and MHD simulation results) often display images of $p B$, at visible wavelengths, the polarization of the F-corona is nearly zero, hence it it not observed in images of $p B$, even at larger distances. But, more importantly, our eyes "see" $B$. Thus, to directly relate our simulation results to the reports of eclipses during the Maunder Minimum, we should limit ourselves to $B$. 


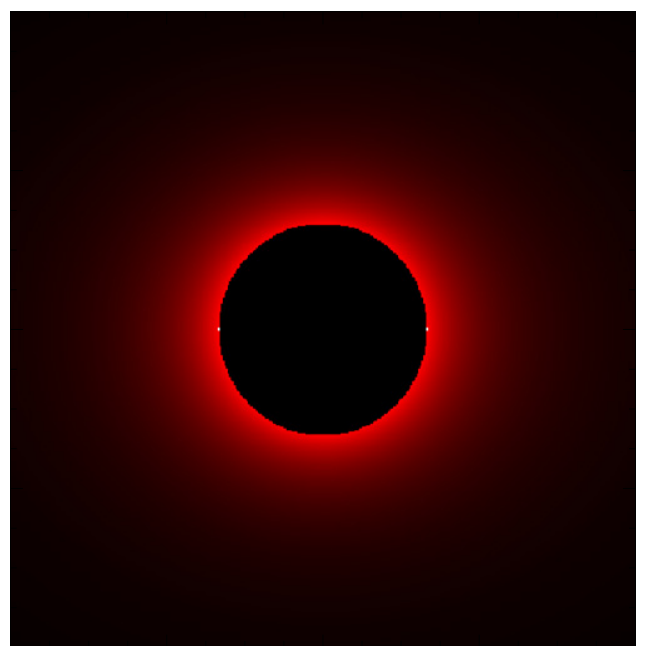

Brightness of F-Corona, B/B

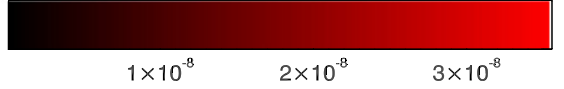

Figure 6. Simulated image of the F-corona, based on the formula by Koutchmy \& Lamy (1985).

Figure 6 is an estimate of the F-corona as it might have looked during the Maunder Minimum using the formulae by Koutchmy \& Lamy (1985). In fact, this picture is indistinguishable from how it would appear today, given that the dust giving rise to it was formed from asteroid collisions and cometary activity, for which the timescales are much longer. We also have taken the liberty of using a red color table based on work suggesting that there is a strong reddening of the spectrum (see Koutchmy \& Lamy (1985) and references therein). It is, however, by no means certain that, if the $\mathrm{K}$ corona disappeared completely, the remaining F-corona would appear as red as shown here. The main point to make is that the F-corona shows no discernible structure with respect to position angle. Although there is a slight variation between the equator and pole, this would be imperceptible to the naked eye. An observer fortunate enough to witness the F-corona directly would report a smooth annulus or halo surrounding the Sun, possibly reddish in color.

To make a direct comparison between the F- and K-coronae during the Maunder Minimum, we computed simulated total brightness $(B)$ images, analogous to those shown in Figure 5. From these, we extracted radial traces taken through the solar equator for each scenario, and compared them with the Fcorona brightness estimates discussed above. These are shown in Figure 7. We remark on the clear separation between scenarios (a)-(d) and (e)-(f). The former dominate over the Fcorona (red) at least out to $2 R_{S}$. The latter, however, are dominated by the F-corona by $1.3 R_{S}$. Comparison with Figure 5 suggests that the range between $1.3 R_{S}$ and $2 R_{S}$ is precisely where coronal structure manifests itself in white-light eclipse observations. Given the idealizations and approximations employed to arrive at this result, it is quite remarkable that such a clear delineation occurs. Based on these results, then, we would anticipate that an eyewitness to scenarios (e) or (f) would not observe any structure of the true $(\mathrm{K}-)$ corona because it would be obscured by the (potentially red) and structureless F-corona. On the other hand, an observer of scenarios (a)-(d) would likely see helmet streamer, pseudo streamer, coronal hole, and plume structure before being washed out by the F-corona.

\section{DISCUSSION}

Our results suggest that the most likely state of the corona during the Maunder Minimum was not merely that of the 2008/ 2009 solar minimum, but rather a state devoid of any largescale structure, driven by a photospheric field composed of only ephemeral regions, and likely substantially reduced in strength. The continued modulation of cosmic rays, including the inferred presence of an $11 \mathrm{yr}$ cycle, as well as albeit modest auroral activity, requires both some magnetic field and a continuing dynamo process. The lack of any observations reporting coronal structure, the possible presence of an Fcorona, and likely decrease in the strength of the IMF also contradict the idea that the Maunder Minimum Sun was no different than in 2008/2009. It is worth noting that Schrijver et al. (2011) and Svalgaard \& Cliver (2007) based their conclusions on assumptions or hypotheses, which were extrapolated back to the Maunder Minimum Interval. Schrijver et al. (2011) argued that there is a minimum state, or "floor" in solar activity, associated with small-scale magnetic bipoles (i.e., what we have called ephemeral regions). However, they did not address whether or not polar coronal holes would have been present during the Maunder Minimum. Svalgaard \& Cliver (2007) posited a similar "floor" but this time, in terms of the strength of the interplanetary magnetic field. This was based on 12 cycles of sunspot data and nuclide data going back to 1500 . The proposed floor of $4.6 \mathrm{nT}$, however, had to be lowered as the 2008/2009 minimum dropped below the value predicted for it.

Our analysis has been limited to qualitative inferences on the likely state of the corona during the Maunder Minimum. However, these are subjective, in the sense that two reasonable people could disagree. For example, while the reports of a "red erie glow" during some of the eclipse observations is suggestive of the presence of an F-corona, one could argue that these were the result of local atmospheric effects, or even sensitivities unique to the observer. In an attempt to incorporate various types of evidence to arrive at a more reliable estimate for the probability that a given hypothesis is true, based on various pieces of evidence, we can invoke a Bayesian type of reasoning. In our case, scenarios (a)-(f) have captured two distinct ideas. First, that the Maunder Minimum Sun was like that in 2008/2009 (the "2008 Sun") ((a)-(d)). And second, that the Sun consisted exclusively of parasitic polarity, with no large-scale dipole component (the "ephemeral-only" Sun, (e)-(f)).

Table 2 lists each piece of evidence that might support either the conclusion that the Maunder Minimum Sun was "2008like" or "ephemeral-like" based on our analysis in Section 2, together with our informed guess about which scenario the evidence supports. If we could derive reasonably accurate likelihood ratios for each piece of evidence listed, we could compute Bayesian estimates of the posterior odds ratio, and, hence, the likelihood of one scenario occurring over another. Unfortunately, we cannot; at best, we could argue that the eclipse observations, for example, make it more likely than not that the Sun was in an ephemeral-only state. On the other hand, with exclusive scenarios, the odds must add to one, so that many pieces of evidence modestly favoring one outcome, at the same time discount the alternative. Thus, we suggest that the 


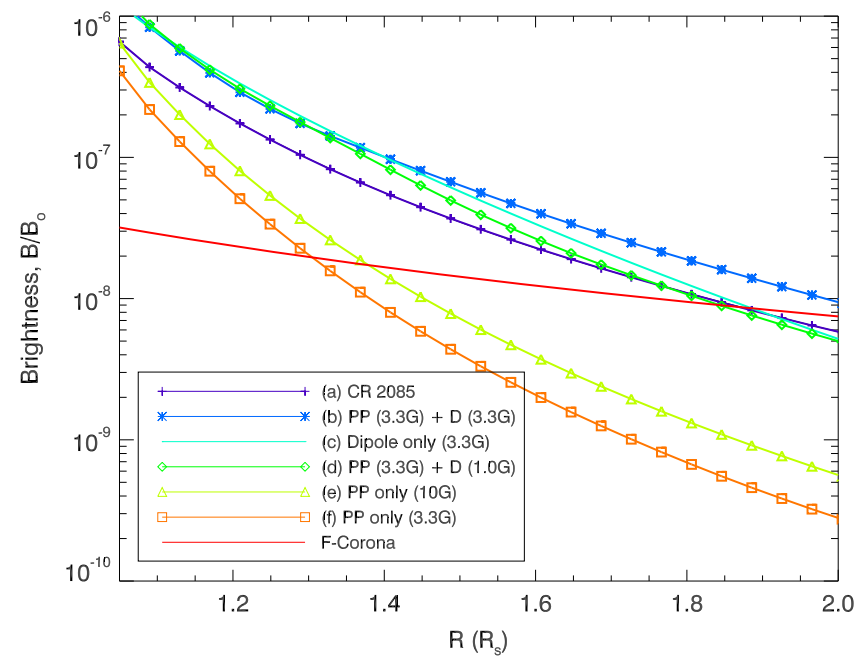

Figure 7. Comparison of radial fall-off in brightness for the six model results shown in Figure 5 with the brightness profile computed for the F-corona (red).

list presented in Table 2 more strongly favors the "ephemerallike" scenario than might be otherwise intuited.

Our invocation of Bayesian methodology to argue that the "ephemeral-only" Sun is strongly favored may seem awkward. In fact, the case for the ephemeral-only Sun can be made simply by scanning Table 2 . However, the Bayesian approach provides two advantages. First, it emphasizes that the individual pieces of evidence combine in a multiplicative fashion to support one hypothesis over the other. Second, it provides a conceptual framework for understanding the fallacy of arguments that seek to promote an alternative hypothesis by attacking the credibility of the evidence supporting the former. For example, consider observations of eclipses during the Maunder Minimum. While one can argue that there may have been omissions or even biases in the reports, this only goes to the credibility of the evidence, that is, a measure of the error bars. The most probable interpretation remains that these observations tend-even if only slightly-to favor the "ephemeral-only" picture, and they actively refute the "2008/ 2009" scenario. As with error analysis in general, we derive the best estimate of the parameter by multiplying the individual parameters together, and then, we add the relative individual errors. We may conclude that the errors are large enough that they admit either hypothesis; however, our conclusion remains that the most likely scenario is the "ephemeral-only" Sun.
Our results and conclusions are sensitive to a number of limitations and assumptions. Not withstanding the quality of the observations and their interpretation, we have applied an MHD code to an interval of time for which we have no experience modeling. The need to invoke a heating mechanism that likely tracks well with large changes in magnetic field strength necessitated a prescription based only on the magnetic field strength. In particular, we excluded an exponential heating term, which we believe is a key component for heating the fast solar wind (Lionello et al. 2009). We did, however, make several test runs with this term on and off, finding that it did not affect the amount of flux opened into the heliosphere in any significant way. Additionally, we recognize that the actual numerical values of the photospheric field strength observed during the space era are not well determined (Riley et al. 2014). However, on the scale of variability we are exploring in this study, such uncertainties are unimportant. More importantly, we have assumed that the heating is roughly proportional to the field strength over (potentially) several orders of magnitude. Although this is apparently borne out by observations across a range of stellar objects (e.g., Fisher et al. 1998; Pevtsov et al. 2003; Riley et al. 2010), it is not necessarily the case that the Sun must adhere to such a rule. Finally, we should note that our limited set of simulations does not preclude a case where the dipole field strength is sufficiently small that it would not conflict with the observations, and particularly the eclipse reports. However, any-even modest-large-scale component is likely to break up the random character of the ephemeral fields, imposing coronal hole structure.

Our study suggests that the so-called "floor" in the strength of the heliospheric magnetic field may have been substantially lower than early estimates suggest (e.g., Svalgaard \& Cliver 2007; Owens et al. 2008). To deduce a revised floor value of the near-Earth IMF strength, Cliver \& Ling (2011) used: (1) a precursor relationship between IMF strength at solar minimum $\left(B_{\min }\right)$ and the subsequent SSN maximum; and (2) a correlation between the solar polar field strength and $B_{\min }$, resulting in a new floor estimate of $\sim 2.8 \mathrm{nT}$. Cliver (2012) suggested that the minimal magnetic state of the Sun characterized by ephemeral active regions identified by Schrijver et al. (2011) could be the source of such a floor. This suggestion is supported by simulation (e) in Table 1 (only ephemeral regions $( \pm 10 \mathrm{G})$, no polar fields) which, using a regression relation between $B_{r}, B$, and $v$ (Cliver \& Ling 2011), produces a field strength of $B=\sim 2.1 \mathrm{nT}$ for an assumed solar wind speed of $\sim 300 \mathrm{~km} \mathrm{~s}^{-1}$.

Table 2

Summary of Observational Evidence for Each of Two Possible Maunder Minimum Scenarios

\begin{tabular}{|c|c|c|c|}
\hline Observation & 2008 Sun & Ephemeral Sun & Notes \\
\hline SSN & $\times$ & $\checkmark$ & Strictly speaking, 2008/2009 cannot represent $\sim 1700$ because sunspots have not been absent for the last 70 yrs \\
\hline Aurora & ? & ? & $\begin{array}{l}\text { Difficult to assess because historical records are not currently maintained, and current measures are limited to } \\
\text { space era }\end{array}$ \\
\hline${ }^{10} \mathrm{Be}$ & $\times$ & $\checkmark$ & $\begin{array}{l}\text { When coupled with neutron monitor measurements and converted to modulation potential, } 2008 \text { was } \\
\text { significantly different than } 1700\end{array}$ \\
\hline${ }^{14} \mathrm{C}$ & $x$ & $\checkmark$ & Unfortunately, natural ${ }^{14} \mathrm{C}$ measurements cannot be extended beyond 1950 \\
\hline Coronal Structure & $\times$ & $\checkmark$ & $\begin{array}{l}\text { Eclipse observations during MM are not unambiguous. However, argument can be made that a } 2008 \text { eclipse } \\
\text { would have been noted }\end{array}$ \\
\hline Red Glow & $x$ & $\checkmark$ & Reports of an "erie" red glow are suggestive of an F-corona (and commensurately fainter, or absent K-corona) \\
\hline 11 yr Periodicity & $\checkmark$ & $x$ & $\begin{array}{l}\text { Continued solar cycle suggests that circulation still proceeding, implying that polar fields continued to build-up } \\
\text { and decay }\end{array}$ \\
\hline
\end{tabular}


A similar relationship in Rouillard et al. (2007) gives $\sim 2.5 \mathrm{nT}$. More recently, Lockwood \& Owens (2011) developed a non-linear relationship between $B$ and $B_{r}$ that gives $B=1.8 \pm 0.6 \mathrm{nT}$ at the end of the Maunder Minimum (Steinhilber et al. 2010).

Many dynamo models of the solar cycle succeed in generating cyclic or pseudo-cyclic variability during Maunder Minimum-like epochs of strongly suppressed activity. In models where the polar fields are generated through turbulent dynamo action within the convection zone, even if the strength of the internal magnetic field were to fall below the sunspot formation threshold, the cycle is actually still running. Cyclic polarity reversals of the (weakened) surface dipole are therefore expected to continue unabated (e.g., Beer et al. 1998). In dynamo models where the surface dipole is generated via the surface decay of active regions, the situation is more delicate, but pseudo-cyclic variability can still be produced during suppressed activity episodes. This can occur either through transport and submergence of slowly decaying residual magnetic fields (e.g., Charbonneau et al. 2004), or intermittent active region emergences and/or secondary turbulent dynamo action (e.g., Passos et al. 2014). Unfortunately, the number of poorly constrained parameters and functionals in most such models precludes any reliable prediction of the strength of the residual surface magnetic field.

An important point to make is that the Maunder Minimum was not a steady period of inactivity. Instead, it is likely that there was a general progression to a progressively deeper configuration during the $70 \mathrm{yr}$ period. Therefore, it may be more reasonable to compare the 2008/2009 solar minimum with the initial descent into the Maunder Minimum; however, it is unlikely that the last 5-10 yrs of the Maunder Minimum looked anything like the recent minimum. Additionally, recent work by Johnsen et al. (2014) suggests that the solar cycle 24 minimum was too brief to reach a true "floor" state, and, in particular, that there remained a significant presence of magnetic flux in the polar regions. Thus, we suggest that the Sun's magnetic field continued to evolve during the Maunder Minimum and posit that this evolution is best represented by an ever-decreasing surface field, and, in particular, the gradual decay of the polar fields. These polar fields are the dominant source of the open flux permeating the heliosphere and modulating the flux of cosmic rays hitting Earth's magnetosphere. By 1700 , the Sun may have consisted of only smallscale parasitic polarity field, with virtually no substantial largescale dipolar component.

Our results are in apparent conflict with several numerical studies. Mackay (2003) used a magnetic flux transport model to conclude that if the grand minimum started at solar cycle minimum, then a large amount of unipolar flux may have persisted in the polar regions of the Sun, whereas, if the minimum had started at solar maximum, there may have been little-to-no large scale magnetic flux on the Sun. Additionally, Wang \& Sheeley (2013) argued that an "ephemeral only" Sun was not possible because the inferred interplanetary magnetic field strength would be inconsistent with estimates based on the ${ }^{10} \mathrm{Be}$ record. Their approach for estimating the open flux that a distribution of randomly orientated small-scale dipoles would produce, however, rested on a magnetostatic extrapolation of the large-scale residual field produced by them. Importantly, it did not take into account the fact that small loops would be heated, expand, and potentially open up into the solar wind- a result that could only be revealed using an MHD approach, as described here. In fact, our results suggest that an ephemeralonly Sun is capable of supplying an open flux that may be $1 / 10$ to $1 / 3$ of the value measured in $2008 / 2009$, and clearly consistent with the cosmogenic records.

In closing, our analysis of the available observations during the Maunder Minimum, together with their interpretation within the context of global MHD model results suggests that this period was unlike anything we have observed in recent times. The cosmogenic record suggests that the interval was not static but continually evolving. We suggest that the period may be bounded by the two states we have compared and contrasted here: that of the 2008/2009 minimum and the ephemeral-only Sun. Moreover, this supports the conjecture by Lockwood (2013) that the Sun may be entering into the next grand minimum.

This work was motivated by discussions during the two ISSI (International Space Science Institute) team workshops on "Long-Term Reconstruction of Solar and Solar Wind Parameters," which occurred in 2012 and 2013, May. PR gratefully acknowledges the support of NASA (Causes and Consequences of the Minimum of Solar Cycle 24 program, LWS Strategic Capabilities program, Heliophysics Theory Program, and the STEREO IMPACT team) and NSF (Center for Integrated Space Weather Modeling (CISM) program). JAL acknowledges NASA's LWS TR\&T program (FST: Using Impulsive SEP Events as Probes of Solar-Heliospheric Structures and the Transport). We gratefully acknowledge support from both NASA's and NSF's supercomputing facilities. Simulation results shown here were completed primarily using NSF's "Stampede."

\section{REFERENCES}

Alanko-Huotari, K., Mursula, K., Usoskin, I. G., \& Kovaltsov, G. A. 2006, SoPh, 238, 391

Athay, R. G. 1986, ApJ, 308, 975

Barnard, L., Lockwood, M., Hapgood, M. A., et al. 2011, GeoRL, 381, L16103 Beer, J., Tobias, S., \& Weiss, N. 1998, SoPh, 181, 237

Berggren, A.-M., Beer, J., Possnert, G., et al. 2009, GeoRL, 36, 11801 Billings, D. E. 1966, A Guide to the Solar Corona (New York: Academic) Charbonneau, P., Blais-Laurier, G., \& St-Jean, C. 2004, ApJL, 616, L183

Clette, F., Svalgaard, L., Vaquero, J. M., \& Cliver, E. W. 2014, SSRv, 1, 35 Cliver, E., \& Ling, A. 2011, SoPh, 274, 285

Cliver, E. W. 2012, in IAU Symp. 286, ed. C. H. Mandrini, \& D. F. Webb, 179 Cliver, E. W., Boriakoff, V., \& Bounar, K. H. 1998, GeoRL, 25, 897

Cowley, S. 1991, AnGeo, 9, 176

Crooker, N. U., \& Owens, M. J. 2010, in ASP Conf. Ser. 428, SOHO-23: Understanding a Peculiar Solar Minimum, ed. S. R. Cranmer, J. T. Hoeksema, \& J. L. Kohl, 279

Eddy, J. A. 1976, Sci, 192, 1189

Fisher, G. H., Longcope, D. W., Metcalf, T. R., \& Pevtsov, A. A. 1998, ApJ, 508,885

Foukal, P., \& Eddy, J. 2007, SoPh, 245, 247

Grant, R. 1852, History of physical astronomy from the earliest ages to the middle of the nineteenth century...The sources of Science (New York: Johnson, 1966) reprint from the London edn

Hoyt, D. V., \& Schatten, K. H. 1996, SoPh, 165, 181

Hoyt, D. V., \& Schatten, K. H. 1998, SoPh, 181, 491

Johnsen, K. D., Kolendersk, P., Scarcella, C., et al. 2014, JAP, 116, 143101

Koutchmy, S., \& Lamy, P. L. 1985, in IAU Coll. 85, Properties and Interactions of Interplanetary Dust, ed. R. H. Giese, \& P. Lamy, 63

Linker, J. A., Mikic, Z., Riley, P., et al. 2012, in AIP Conf. Proc. 1539, Proc. Solar Wind 13 ed. G. P. Zank, J. Borovsky, R. Bruno et al. (Melville, NY: AIP) , 26

Lionello, R., Linker, J. A., \& Mikić, Z. 2009, ApJ, 690, 902

Lockwood, M. 2013, LRSP, 10, 4

Lockwood, M., Nevanlinna, H., Barnard, L., et al. 2014a, AnGeo, 32, 383 
Lockwood, M., Nevanlinna, H., Vokhmyanin, M., et al. 2014b, AnGeo, 32, 367

Lockwood, M., \& Owens, M. 2014, JGRA, 119, 5193

Lockwood, M., Owens, M., \& Rouillard, A. 2009, JGRA, 114, A11104

Lockwood, M., \& Owens, M. J. 2011, JGR, 116, 4109

Lockwood, M., Owens, M. J., \& Barnard, L. 2014a, JGRA, 119, 5172

Lockwood, M., Owens, M. J., \& Barnard, L. 2014b, JGRA, 119, 5183

Mackay, D. H. 2003, SoPh, 213, 173

Maunder, E. W. 1894, Knowledge, 17, 173

McCracken, K., \& Beer, J. 2014, JGRA, 119, 2379

McCracken, K., Beer, J., Steinhilber, F., \& Abreu, J. 2013, SSRv, 176, 59

McCracken, K. G., \& Beer, J. 2007, JGRA, 112, 10101

McCracken, K. G., Beer, J., \& McDonald, F. B. 2005, ISSIR, 3, 83

McCracken, K. G., McDonald, F. B., Beer, J., Raisbeck, G., \& Yiou, F. 2004, JGRA, 109, 12103

Mikić, Z., Linker, J. A., Lionello, R., Riley, P., \& Titov, V. 2007, in ASP Conf. Ser. 370, Solar and Stellar Physics Through Eclipses, ed. O. Demircan, S. O. Selam, \& B. Albayrak, 299

Miyahara, H., Masuda, K., Muraki, Y., et al. 2004, SoPh, 224, 317

Owens, M., Usoskin, I., \& Lockwood, M. 2012, GeoRL, 39, L19102

Owens, M. J., \& Crooker, N. U. 2006, JGR, 111, 10104

Owens, M. J., Crooker, N. U., Schwadron, N. A., et al. 2008, GeoRL, 35, 20108

Parker, E. N. 1976, in IAU Symp. 71, Basic Mechanisms of Solar Activity, ed. V. Bumba, \& J. Kleczek, 3

Passos, D., Nandy, D., Hazra, S., \& Lopes, I. 2014, A\&A, 563, A18

Pevtsov, A. A., Fisher, G. H., Acton, L. W., et al. 2003, Astrophys. J., 598, 1387

Reimer, P. J., Baillie, M. G. L., Bard, E., et al. 2004, Radiocarbon, 46, 1029

Réthly, A., \& Berkes, Z. 1963, Nordlichtbeobachtungen in Ungarn (Verlag der Ungarischen Akademie der Wissenschaften)

Ribes, J., \& Nesme-Ribes, E. 1993, A\&A, 276, 549

Riley, P. 2010, in AIP Conf. Proc. 1216, 12th Int. Solar Wind Conf., ed. M. Maksimovic, K. Issautier, N. Meyer-Vernet, M. Moncuquet, \& F. Pantellini, 323

Riley, P., Ben-Nun, M., Linker, J. A., et al. 2014, SoPh, 289, 769

Riley, P., Linker, J. A., Lionello, R., \& Mikic, Z. 2012, JASTP, 83, 1

Riley, P., Linker, J. A., \& Mikić, Z. 2001, JGR, 106, 15889
Riley, P., Lionello, R., Linker, J. A., et al. 2011, SoPh, 274, 361

Riley, P., Lionello, R., Mikić, Z., et al. 2007, ApJ, 655, 591

Riley, P., \& Luhmann, J. G. 2012, SoPh, 277, 355

Riley, P., Mikic, Z., Lionello, R., et al. 2010, JGR, 115, 6104

Rouillard, A., Lockwood, M., \& Finch, I. 2007, JGRA, 112, A05103

Schrijver, C. J., Livingston, W. C., Woods, T. N., \& Mewaldt, R. A. 2011, GeoRL, 38, 6701

Schröder, W. 1992, JGG, 44, 119

Schwadron, N. A., Connick, D. E., \& Smith, C. 2010, ApJL, 722, L132

Silverman, S. 1992, RvGeo, 30, 333

Siscoe, G. L. 1980, RvGSP, 18, 647

Smith, E. J., \& Balogh, A. 2008, GeoRL, 35, 22103

Smith, E. J., \& Marsden, R. G. 2003, GeoRL, 30, 1

Solanki, S. K., Usoskin, I. G., Kromer, B., Schüssler, M., \& Beer, J. 2004, Natur, 431, 1084

Sporer, E. 1887, Vierteljahrsschr. Astron. Ges. Leipzig, 22, 323

Steinhilber, F., Abreu, J., Beer, J., \& McCracken, K. 2010, JGRA, 115, A01104

Steinhilber, F., Abreu, J. A., Beer, J., et al. 2012, PNAS, 109, 5967

Stevens, M., Linker, J. A., \& Riley, P. 2012, JASTP, 83, 22

Suess, H. E. 1955, Sci, 122, 415

Suess, S. T. 1979, P\&SS, 27, 1001

Svalgaard, L., \& Cliver, E. W. 2007, ApJL, 661, 203

Usoskin, I., Hulot, G., Gallet, Y., et al. 2014, A\&A, 562, L10

Usoskin, I. G. 2008, LRSP, 5, 3

Usoskin, I. G., Bazilevskaya, G. A., \& Kovaltsov, G. A. 2011, JGRA, 116,2104

Usoskin, I. G., Mursula, K., \& Kovaltsov, G. A. 2001, JGRA, 106, 16039

Usoskin, I. G., Solanki, S. K., Schüssler, M., Mursula, K., \& Alanko, K. 2003, PhRvL, 91, 211101

Wang, Y.-M., \& Sheeley, N. R. 1995, ApJL, 447, L143

Wang, Y.-M., \& Sheeley, N. R., Jr. 2013, ApJ, 764, 90

Wing, V. 1656, Astronomica Instaurata, Or, A New and Compendious Restauration of Astronomie: In Four Parts ... Whereunto is Added, a Short Catalogue of All the Most Accurate and Remarkable Coelestiall Observations, that Have Been Made by Tycho, Longomontanus, Gassendus, the Landgrave of Hassia, and Others (R. and W. Leybourn) 